\title{
An Analytical Solution for Transient Thermal Response of an Insulated Structure
}

\author{
Max L. Blosser* \\ NASA Langley Research Center, 23681, USA
}

\begin{abstract}
An analytical solution was derived for the transient response of an insulated aerospace vehicle structure subjected to a simplified heat pulse. This simplified problem approximates the thermal response of a thermal protection system of an atmospheric entry vehicle. The exact analytical solution is solely a function of two non-dimensional parameters. A simpler function of these two parameters was developed to approximate the maximum structural temperature over a wide range of parameter values. Techniques were developed to choose constant, effective properties to represent the relevant temperature and pressure-dependent properties for the insulator and structure. A technique was also developed to map a time-varying surface temperature history to an equivalent square heat pulse. Using these techniques, the maximum structural temperature rise was calculated using the analytical solutions and shown to typically agree with finite element simulations within 10 to 20 percent over the relevant range of parameters studied.
\end{abstract}

\section{Nomenclature}

$a_{1}, a_{2}$ Coefficients of approximate equation for maximum structural temperature

$b_{n} \quad$ Coefficients of series solution for $\tau>\tau_{h}$

$c_{m} \quad$ Coefficients of series solution for $0<\tau \leq \tau_{h}$

$c_{p e} \quad$ Effective insulator specific heat capacity

$c_{p s} \quad$ Structural specific heat capacity

$d_{e} \quad$ Insulator thickness

$d_{s} \quad$ Structural thickness

$k_{e} \quad$ Effective insulator thermal conductivity

$P_{\text {avg }} \quad$ Average ambient pressure during equivalent square surface temperature pulse

$T$ Temperature

$t \quad$ Time

$T_{h} \quad$ Applied surface temperature rise of insulator

$t_{h} \quad$ Duration of heating pulse

$T_{i} \quad$ Initial temperature

$T_{m} \quad$ Maximum structural temperature rise

$T_{c e} \quad$ Temperature to use for calculating effective insulation specific heat capacity

$T_{c s} \quad$ Temperature to use for calculating effective structural specific heat capacity

$T_{k e} \quad$ Temperature to use for calculating effective insulation conductivity

$x \quad$ Non-dimensionalized spatial variable

$x^{\prime} \quad$ Spatial variable, position through insulator thickness

Symbols

$\beta \quad$ Ratio of insulator conductance to insulator heat capacitance

$\gamma \quad$ Ratio of insulator to structural heat capacitance

$\lambda \quad$ Eigenvalue

$\rho_{e} \quad$ Effective insulator density

*Research Engineer, Structural Mechanics and Concepts Branch 


$\begin{array}{ll}\rho_{s} & \text { Structural density } \\ \tau & \text { Non-dimensionalized time } \\ \tau_{h} & \text { Non-dimensionalized duration of heat pulse } \\ \tau_{m} & \text { Non-dimensionalized time of maximum structural temperature } \\ \theta & \text { Non-dimensionalized temperature }\end{array}$

\section{Introduction}

$\mathrm{T}$

HERMAL protection systems are a critical component of hypersonic and atmospheric entry vehicles. The reusable ceramic tiles and blankets of the Space Shuttle Orbiter work very well as thermal insulators, but result in a fragile, high maintenance exterior surface. An intriguing approach to this problem is to build the thermal insulation into the exterior vehicle wall. This deceptively simple idea will be difficult to achieve because it requires a flight weight aerospace vehicle skin to not only carry the required mechanical loads, but also to accommodate severe transient heating with the corresponding hot outer surface and large temperature gradients through its thickness.

A thermally insulating structural panel will likely be of a sandwich construction as a result of both thermal and structural considerations. The outer, heated face sheet of the sandwich will typically be a thin layer of non-insulating material that will contribute very little to the thermal response of the inner, unheated face sheet. Thus, ignoring edge closeouts and joints, the transient thermal response of an insulating sandwich panel will be similar in character to the thermal response of an insulated structure like the external tiles and blankets covering the aluminum structure of the Space Shuttle Orbiter. For a non-homogeneous sandwich core, effective thermal properties may be calculated using a rule of mixtures to approximate its thermal performance.

In this paper a simplified transient thermal problem was investigated in an attempt to gain basic insight that will be required to develop optimum sandwich panels that can simultaneously insulate and carry structural loads. An analytical solution is derived for the transient response of an insulated structure to a simplified heat pulse. The solution is a function of two non-dimensional quantities. A simpler function of these two parameters is developed to approximate the maximum structural temperature over a wide range of parameter values. Techniques were developed to choose constant, effective properties to represent the relevant temperature and time-dependent properties for the insulator and structure. A technique was also developed to map a time-varying surface temperature history to an equivalent square heat pulse. Using these techniques, the maximum structural temperature rise was calculated using the analytical solutions and compared with finite element simulations over a wide range of parameters. Results for the analytical series solution were typically within 10 to 20 percent of the finite element solutions. The approximate analytical solution had similar accuracy for many of the cases studied, but began to lose accuracy as one of the non-dimensional governing parameters became small.

\section{Problem Definition}

The simplified problem investigated in this paper is illustrated in Figure 1. A thermal insulator with thickness, $d_{e}$, density, $\rho_{e}$, specific heat capacity, $c_{p e}$, and thermal conductivity, $k_{e}$ covers a structure (inner face sheet) of thickness, $d_{s}$, density, $\rho_{s}$, and specific heat capacity, $c_{p s}$. The inner surface of the structure is assumed to be perfectly insulated to simplify the mathematics of the problem and because it is a commonly used conservative assumption for sizing thermal protection systems. To further simplify the problem, the structure is treated as a lumped heat capacitance and the outer face sheet of the insulating sandwich panel is neglected. For this solution the material properties are assumed to be constant, so effective, averaged properties would have to be used to approximate more complex material behavior.

A simple transient heat pulse was assumed. Initially the insulator and structure are assumed to be at a uniform temperature of 0 . The outer surface of the insulator is assumed to instantaneously rise to a temperature $T_{h}$ at $t=0$ and maintain that temperature until $t=t_{h}$ at which time it instantaneously returns to 0 . 


\section{II.A. Mathematical Description}

Using a non-dimensional spatial variable, $x=\frac{x^{\prime}}{d_{e}}$, the governing differential equation for heat conduction through the insulator can be written as

$$
\frac{\partial T}{\partial t}=\beta \frac{\partial^{2} T}{\partial^{2} x}
$$

where

$$
\beta=\frac{k_{e}}{\rho_{e} c_{p e} d_{e}^{2}}
$$

The boundary condition at $x=1$ is defined as

$$
\frac{\partial T(1, t)}{\partial t}=-\beta \gamma \frac{\partial T(1, t)}{\partial x}
$$

where

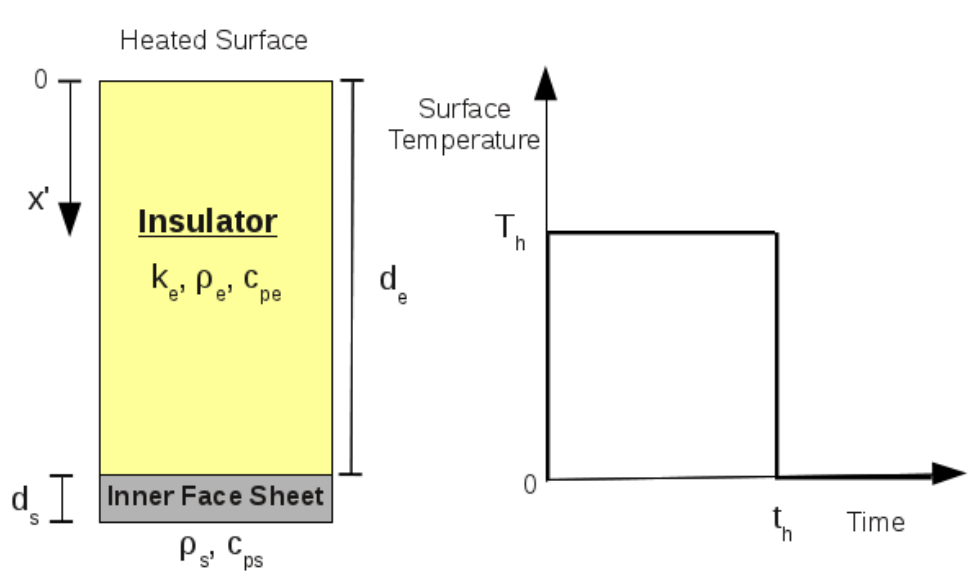

Figure 1. Illustration of simplified problem

$$
\gamma=\frac{\rho_{e} c_{p e} d_{e}}{\rho_{s} c_{p s} d_{s}}
$$

The boundary condition at $x=0$ is defined as

$$
T(0, t)= \begin{cases}T_{h} & \text { for } 0<t \leq t_{h} \\ 0 & \text { for } t>t_{h}\end{cases}
$$

Finally, the initial condition is a uniform temperature of 0 .

$$
T(x, 0)=0
$$

The mathematical problem defined by Equations 1 through 6 can be completely non-dimensionalized by defining a non-dimensional time, $\tau=\beta t$ and a non-dimensional temperature, $\theta=\frac{T}{T_{h}}$. The nondimensionalized differential equation becomes

$$
\frac{\partial \theta}{\partial \tau}=\frac{\partial^{2} \theta}{\partial^{2} x}
$$

The boundary condition at $x=1$ becomes

$$
\frac{\partial \theta(1, \tau)}{\partial \tau}=-\gamma \frac{\partial \theta(1, \tau)}{\partial x}
$$

The boundary condition at $x=0$ becomes

$$
\theta(0, \tau)= \begin{cases}1 & \text { for } 0<\tau \leq \tau_{h} \\ 0 & \text { for } \tau>\tau_{h}\end{cases}
$$

where

$$
\tau_{h}=\frac{k_{e}}{\rho_{e} c_{p e} d_{e}^{2}} t_{h}
$$

The initial condition is

$$
\theta(x, 0)=0
$$




\section{II.B. Non-dimensional Governing Parameters}

Inspection of the mathematical problem defined by Equations 7 through 11 reveals that any solution will be completely governed by two non-dimensional parameters, $\gamma$ and $\tau_{h}$. Each of these parameters has clear physical significance. The first parameter, $\gamma$, is defined by Equation 4 which shows it to be the heat capacitance of the insulator divided by the heat capacitance of the underlying structure.

Equation 10 defining the second parameter, $\tau_{h}$, can be slightly rewritten to show that it is the ratio of insulator conductance to heat capacitance times the duration of the heat pulse. The same ratio of insulator conductance to heat capacitance, $\beta$, is used to non-dimensionalize time.

$$
\tau_{h}=\frac{\frac{k_{e}}{d_{e}}}{\rho_{e} c_{p e} d_{e}} t_{h}
$$

\section{Analytical Solution}

An analytical solution for the problem illustrated in Figure 1 can be obtained by combining two existing solutions with modifications. The solution of the first part of the problem, $0<t \leq t_{h}$, can be found in the classic heat transfer textbook by Carslaw and Yeager. ${ }^{2}$ Converting the solution to the nomenclature used in this paper and non-dimensionalizing produces:

$$
\theta(x, \tau)=\frac{T(x, \tau)}{T_{h}}=1-\sum_{m=1}^{\infty} c_{m} \sin \left(\lambda_{m} x\right) e^{-\lambda_{m}^{2} \tau}
$$

where

$$
c_{m}=\frac{2\left(\lambda_{m}^{2}+\gamma^{2}\right)}{\lambda_{m}\left(\lambda_{m}^{2}+\gamma^{2}+\gamma\right)}
$$

The values for $\lambda_{m}$ can be found by solving the equation:

$$
\lambda_{m} \tan \lambda_{m}=\gamma
$$

Solving the second part of the problem, $t>t_{h}$, requires more effort. There is an existing solution for a similar problem in which the insulator and lumped mass are initially at a uniform temperature, but at $t=0$ the temperature of the insulator outer surface is instantaneously reduced to 0 . A solution to this similar problem, including its derivation, was developed by De Chant. ${ }^{3}$ A solution to the second part of the current problem is obtained by closely following the approach used by De Chant, however, instead of a uniform temperature, the solution to Equation 13 at time $t_{h}$ is used as the initial temperature distribution. Details of the derivation are documented in a separate paper. ${ }^{4}$

So, for $t>t_{h}$ or $\tau>\tau_{h}$ the solution is:

$$
\theta(x, \tau)=\frac{T(x, \tau)}{T_{h}}=\sum_{n=1}^{\infty} b_{n} \sin \left(\lambda_{n} x\right) e^{-\lambda_{n}^{2}\left(\tau-\tau_{h}\right)}
$$

where

$$
b_{n}=c_{n}\left(1-e^{-\lambda_{n}^{2} \tau_{h}}\right)-\sum_{m \neq n, m=1}^{\infty}\left(\frac{\frac{\sin \left(\lambda_{m}-\lambda_{n}\right)}{\left(\lambda_{m}-\lambda_{n}\right)}-\frac{\sin \left(\lambda_{m}+\lambda_{n}\right)}{\left(\lambda_{m}+\lambda_{n}\right)}+\frac{2 \sin \lambda_{m} \sin \lambda_{n}}{\gamma}}{1-\frac{\sin \left(2 \lambda_{n}\right)}{2 \lambda_{n}}+\frac{2 \sin ^{2} \lambda_{n}}{\gamma}}\right) c_{m} e^{\left(-\lambda_{m}^{2} \tau_{h}\right)}
$$

The quantities $c_{m}$ and $c_{n}$ can be calculated using Equation 14 and values for $\lambda_{m}$ and $\lambda_{n}$ are obtained by solving Equation 15. In Equation 17 the summation excludes the term $m=n$ because the contribution of that term is captured by the exponential term preceding the summation. 


\section{Numerical Example for Series Solution}

The series solution described by Equations 13 and 16 can be applied to a wide range of physical situations. However, the motivation for deriving the solution was to gain insight into the thermal response of an insulated structure of an aerospace vehicle subjected to a transient aerodynamic heating pulse. Therefore a numerical example was chosen to represent a typical location on the Space Shuttle Orbiter at which the aluminum structure is protected from aerodynamic heating by an LI-900 ceramic tile. The material properties, ${ }^{5}$ are listed in Table 1 along with dimensions, heating duration and the corresponding non-dimensional parameters. Thermal properties are temperature dependent, so the properties for the aluminum structure are for $200^{\circ} \mathrm{F}$ and the properties for the LI-900 tile are for $1250^{\circ} \mathrm{F}$. These temperatures are typical of the average temperatures the insulator and structure might experience in flight. The LI-900 thermal conductivity is also a strong function of pressure, so the conductivity is chosen for a pressure of $0.01 \mathrm{~atm}$ (an arbitrary value in the mid-range of a typical atmospheric pressure profile).

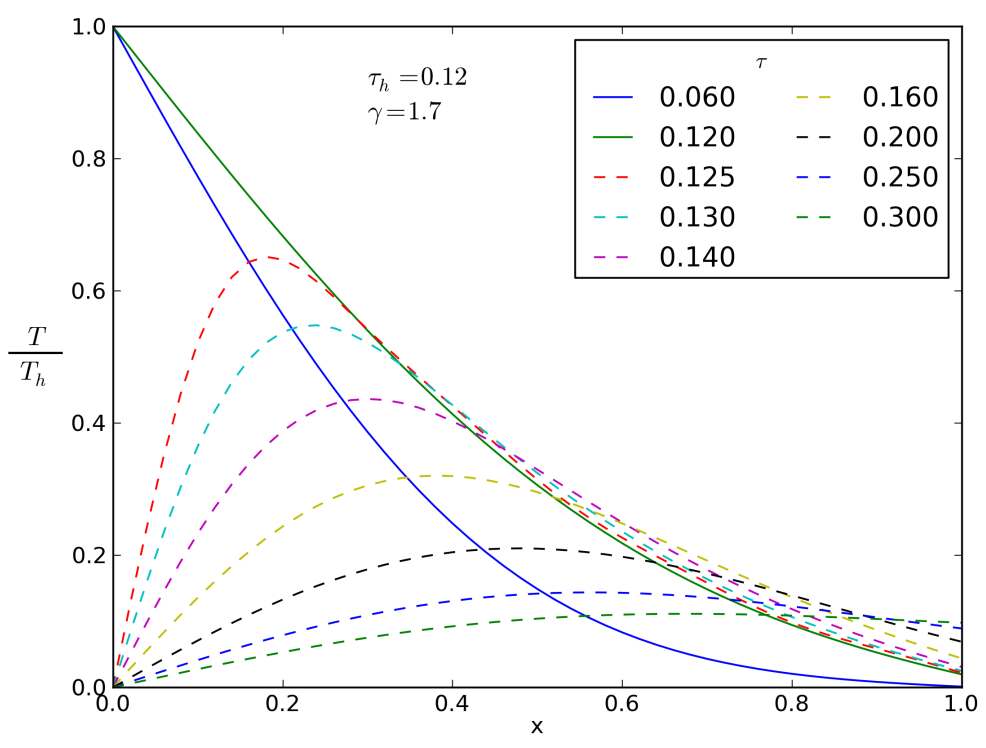

Figure 2. Temperature distributions for 3 inch thick tile
A computer program was written using Version 2.7 of the Python programming language to implement the series solution described by Equations 13 and 16. Routines from Version 0.9 of the SciPy programming library ${ }^{6}$ were used for numerical solutions of nonlinear equations and fitting coefficients to nonlinear equations. For times just after an instantaneous change in surface temperature many terms of the series solution were required for an results shown in Figures 2 and 3, three terms were used for the coefficients $c_{m}$ and eight terms for $b_{n}$. Use of additional terms produced negligible changes in the results. To generate results for the wide range of parameter values shown in Figures 4 , and 5 , twelve terms each were used for $b_{n}$ and $c_{m}$ to reduce the chance of truncation error. accurate solution. However, for the

The non-dimensional temperature distributions through the thickness of the insulator at several times are shown in Figure 2 for a LI-900 tile thickness of 3 inches. The solid blue line represents the temperature distribution halfway through the heat pulse and the solid green line represents the temperature at the end of the heat pulse, $\tau=\tau_{h}$. The dashed lines represent temperature distributions after the heat pulse has ended, $\tau>\tau_{h}$.

After the tile surface temperature drops back to the initial temperature, the heat stored in the tile interior begins to be conducted back out of the cool tile surface. However, the underlying structure, $\mathrm{x}=1$, continues to increase in temperature until its temperature matches that of the adjacent tile, then it begins to cool. The dashed lines in Figure 2 only illustrate the behavior up to the time when the structural temperature has nearly reached that of the adjacent insulator. The next figure will illustrate the time dependent behavior of the structure more fully. This behavior is consistent with the assumption that the structure is perfectly insulated on its inner surface. 
For sizing of thermal protection systems, the temperature of the underlying structure is of primary concern. Further insight into the solution can be obtained by calculating the structural temperature as a function of time. Figure 3 shows a number of temperature histories for different combinations of the governing nondimensional parameters. In Figure 3, the blue lines represent the structural temperature histories for $\tau_{h}=0.05$, the green $\tau_{h}=0.15$, and the red $\tau_{h}=0.3$. The vertical dotted lines represent the end of the heat pulse for the respective values of $\tau_{h}$. The solid, dashed and dash-dot lines represent values of $\gamma$ of 1,2 , and 3 respectively. Because the non-dimensionalized time axis makes the results more difficult to interpret physically, it is helpful to consider the case of fixed material properties and heating duration. The

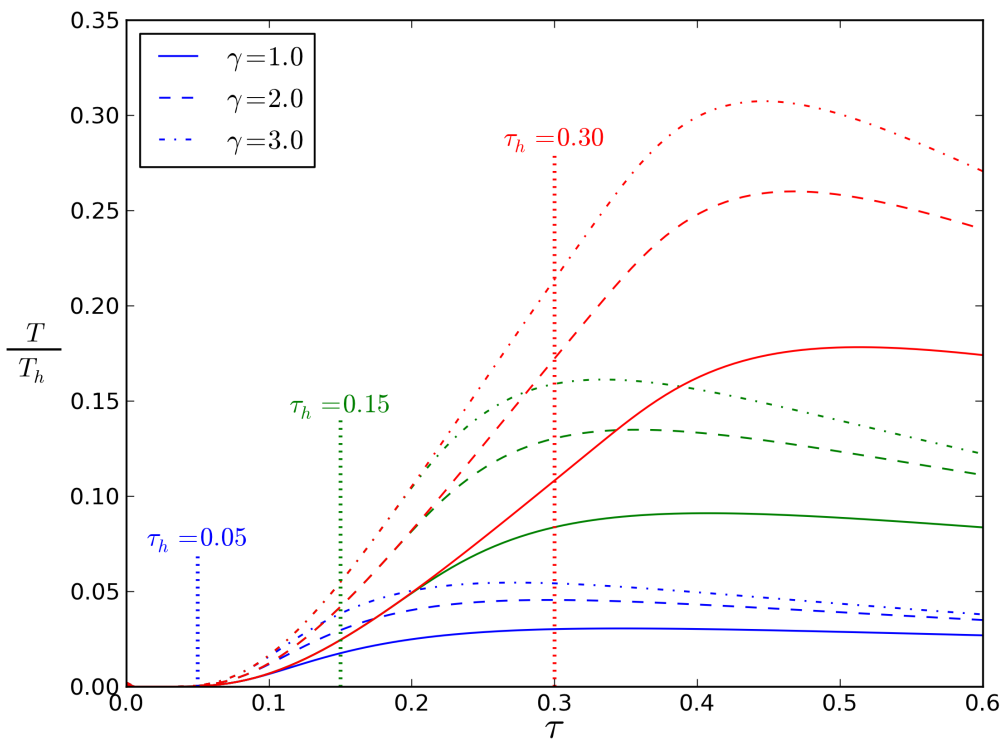

Figure 3. Structural temperature histories as a function of governing parameters

remaining free parameters would be the insulator thickness, $d_{e}$, and the structural thickness, $d_{s}$. The parameter $\tau_{h}$ varies as $\frac{1}{d_{e}^{2}}$, so smaller values of $\tau_{h}$ imply larger values of $d_{e}$. Choosing a value of $\tau_{h}$ fixes the insulator thickness, so for a specified value of $\tau_{h}, \gamma$ can only be varied by changing the structural thickness. The parameter $\gamma$ varies as $\frac{1}{d_{s}}$ so larger values of $\gamma$ imply smaller structural thicknesses. In Figure 3 the structural temperature histories for $\tau_{h}=0.05$ stay much cooler, which is consistent with the expected behavior for thicker insulation. Conversely the structural temperatures reach much higher values for $\tau_{h}=0.3$, which is consistent with the expected behavior for thinner insulation. For each value of $\tau_{h}$ the curve for $\gamma=1$ reaches the lowest maximum temperature, as expected for the correspondingly highest structural heat capacity. Higher $\gamma$ 's result in higher maximum structural temperatures as expected for the associated reduction in structural heat capacity.

All of the structural temperature history curves in Figure 3 exhibit similar behavior. The temperature of the structure continues to rise well after completion of the heating pulse, reaches a maximum value, and then begins to decrease. The maximum temperature is a primary design driver for sizing of thermal protection systems, so it is desirable to be able to readily calculate the maximum structural temperature.

\section{Maximum Structural Temperature}

All of the temperature histories, away from the heated surface, generated using Equation 16 would appear to heat up, reach a maximum temperature, and then begin to cool. The time at which the maximum temperature occurs can be found by taking the first derivative with respect to time, setting it equal to zero, and solving for time. This results in the following equation to be solved for $\tau_{m}$ :

$$
0=-\sum_{n=1}^{\infty} \lambda_{n}^{2} b_{n} \sin \left(\lambda_{n} x\right) e^{-\lambda_{n}^{2}\left(\tau_{m}-\tau_{h}\right)}
$$

Equation 18 can be solved for the time of the maximum temperature for any location through the thickness of the insulator. However, for the current problem, the maximum structural temperature at $x=1$ is of greatest interest. The equation therefore becomes:

$$
0=-\sum_{n=1}^{\infty} \lambda_{n}^{2} b_{n} \sin \left(\lambda_{n}\right) e^{-\lambda_{n}^{2}\left(\tau_{m}-\tau_{h}\right)}
$$


Although Equation 19 cannot be readily solved for $\tau_{m}$ in closed form, it can be solved numerically. The resulting $\tau_{m}$ can be substituted into Equation 16 to obtain the maximum structural temperature. Following this procedure, the maximum structural temperature was calculated over a range of the governing non-dimensional parameters $(0.2 \leq \gamma \leq 5$ and $\left.0.02 \leq \tau_{h} \leq 0.5\right)$. Figure 4 shows a surface plot of the maximum structural temperatures calculated over a 49 by 49 point grid. To gain insight into the physical implications of this plot it is again helpful to consider the case of fixed material properties. For fixed properties, small values of $\tau_{h}$ imply a short heat pulse and/or thick insulator and large values imply a long heat pulse and/or thin insulator. It is logical to expect that as the heat pulse duration goes to zero, $\tau_{h} \rightarrow 0$, the maximum structural temperature will also go to zero. The parameter, $\gamma$, is the ratio of insulation to structural

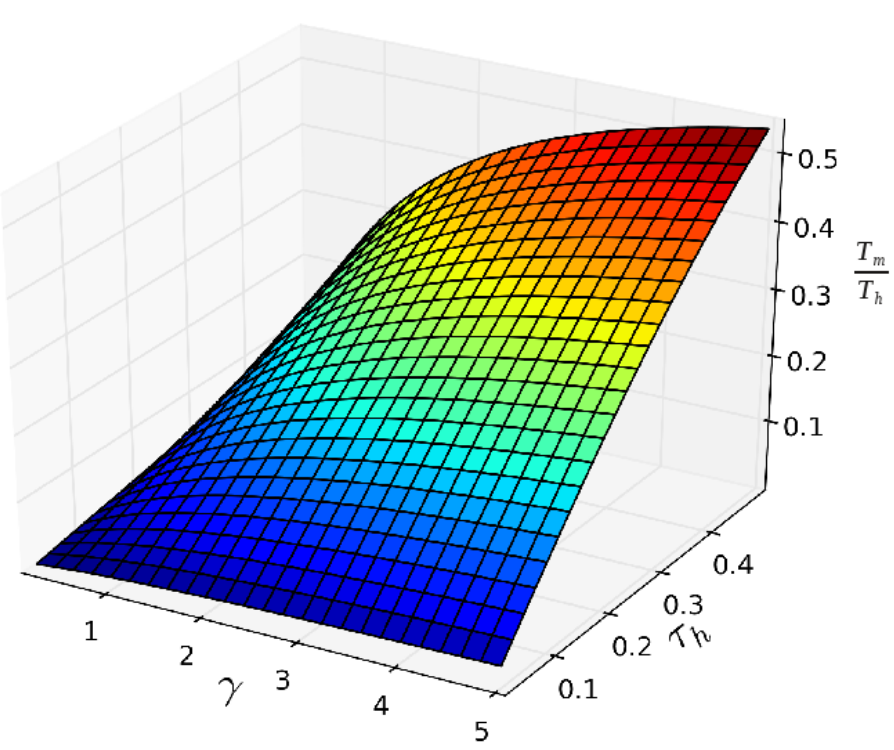

Figure 4. Maximum structural temperatures heat capacitance, so small values imply that the structural heat capacitance is much larger than that of the insulation and large values imply a relatively small amount of structural heat capacitance. Therefore, as the structural heat sink increases towards infinity, $\gamma$ will approach 0 and, for a finite heating duration, the maximum structural temperature will also go to zero. Also, for this problem, the maximum structural temperature cannot exceed the applied surface temperature, so $\frac{T_{m}}{T_{h}} \leq 1$.

The maximum structural temperature plot in Figure 4 is useful to illustrate how the maximum structural temperature varies with the two governing parameters, but it was generated using a complicated series of numerical solutions. For quick calculations, it would be much more useful to have a relatively simple algebraic equation to approximate this surface. The approximate solution should go to zero if either of the governing

Table 2. Coefficient values for Equation 20

\begin{tabular}{cc}
\hline Coefficient & Value \\
\hline$a_{1}$ & -0.72058 \\
$a_{2}$ & 0.53649
\end{tabular}
parameters goes to zero, and it should approach 1 as either parameter goes to infinity. A number of candidate equations were evaluated and the following approximate solution was chosen as a good compromise between simplicity and accuracy:

$$
\left(\frac{T_{m}}{T_{h}}\right)_{a}=1-e^{\left(a_{1} \gamma^{a_{2}} \tau_{h}{ }^{2 a_{2}}\right)}
$$

A "least squares" routine was used to find the values of the coefficients in Equation 20 that best approximate the surface shown in Figure 4. The coefficient values are given in Table 2.

Inspection of Table 2 reveals that $a_{1} \approx \frac{-1}{\sqrt{2}}$ and $a_{2} \approx \frac{1}{2}$. Substituting these coefficients into Equation 20 simplifies the equation to:

$$
\left(\frac{T_{m}}{T_{h}}\right)_{a}=1-e^{-\tau_{h} \sqrt{\frac{\gamma}{2}}}
$$

The relative errors between the approximation of Equation 20 and the series solution were calculated using the following equation:

$$
\epsilon_{r}=\frac{\left(\frac{T_{m}}{T_{h}}\right)_{a}-\frac{T_{m}}{T_{h}}}{\frac{T_{m}}{T_{h}}}
$$


Figure 5 shows the distribution of the relative error over the previously chosen range of governing parameters. Over most of the parameter space, the relative error is well below \pm 10 percent. For small values of $\gamma$, however, the relative error rises precipitately. Therefore it would be prudent to check the values of $\gamma$ for any calculations made using Equation 20 to avoid the inaccurate region. This inaccurate region of the approximate solution would correspond to a physical situation where the structure is much heavier than the insulator.

\section{Comparison of Analytical and Numerical Solutions}

The problem illustrated in Figure 1 is considerably simpler than a typical numerical simulation of aerodynamic heating of an insulated aerospace vehicle structure. Material prop-

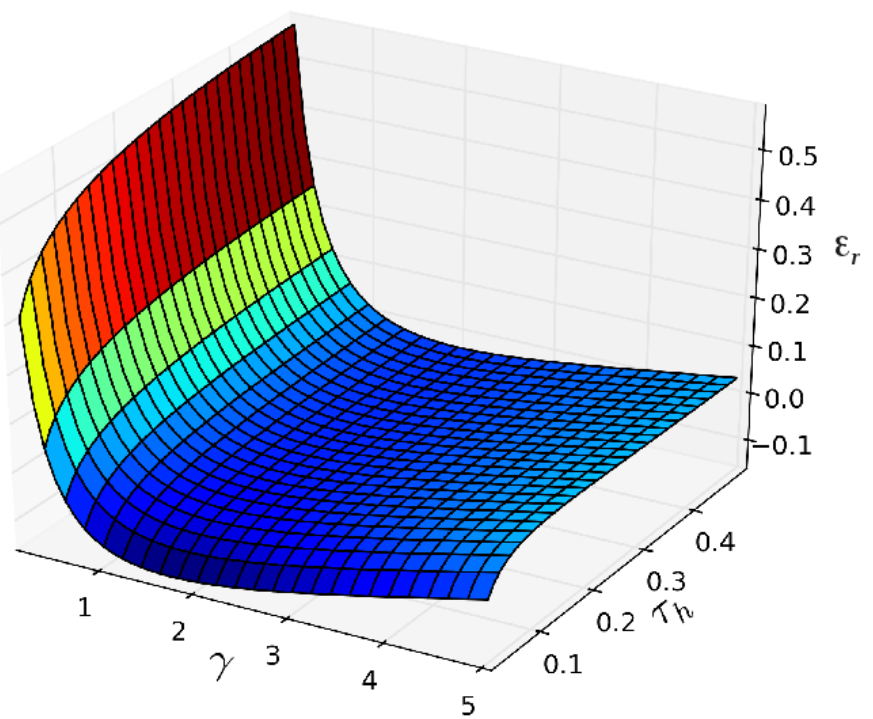

Figure 5. Relative errors using approximate maximum structural temperature equation erties, which can vary with temperature and pressure, are treated as constant. The applied surface temperature is a simple square pulse, rather than a more realistic transient profile. A numerical model was developed to: 1) demonstrate that the series solution, Equations 13 and 16, produce the expected results, 2) verify that the governing nondimensional parameters $\left(\gamma\right.$ and $\tau_{h}$ ), rather than individual material property values, determine the calculated temperatures, 3) develop techniques for best using the analytical solution to approximate a realistic numerical simulation, and 4) estimate typical errors involved in using the analytical solution to approximate a realistic numerical simulation.

\section{VI.A. Numerical Model}

A one-dimensional finite element model was developed using the DOLFIN ${ }^{7}$ finite element library for the Python programming language. Using the DOLFIN library, linear one dimensional elements were used to discretize the spatial dimension and an implicit Crank-Nickelson time marching scheme was used to solve the weak formulation of the diffusion equation. The model consisted of a layer of insulator material in perfect contact with a layer of structural material similar to the configuration shown in Figure 1, except that the structure in the finite element model was not treated as a lumped heat capacitance. For the results shown in this paper, the model consisted of 50 elements through the thickness of the insulator and 2 elements through the thickness of the structure. The boundary condition on the outer surface of the insulator consisted of an imposed surface temperature that could be varied arbitrarily with time and updated at each time step of the solution. The inner surface of the structure was adiabatic. The material properties could be arbitrary functions of temperature and ambient pressure. Material property values for each finite element were updated at each time step of the analysis. Property values could be different for each element, but did not vary spatially within an element. Time steps between 1 and 5 seconds were used to calculate the results presented in this paper.

\section{VI.B. Approximation of Realistic Simulations}

To compare the maximum structural temperature from the analytical solutions to that calculated using a full numerical simulation, it is necessary to choose effective constant properties to use in the analytical solutions. Effective constant material properties were chosen ${ }^{4}$ as follows:

$$
c_{p s}=c_{p s}\left(T_{c s}\right), \text { where } T_{c s}=T_{i}+\frac{T_{m}}{2},
$$




$$
\begin{gathered}
c_{p e}=c_{p e}\left(T_{c e}\right), \text { where } T_{c e}=T_{i}+T_{m}, \text { and } \\
k_{e}=k_{e}\left(T_{k e}, P_{a v g}\right), \text { where } T_{k e}=T_{i}+0.6 T_{h} .
\end{gathered}
$$

To use the analytical solution required defining an equivalent square heating pulse, $t_{h}$ and $T_{h}$, that represents a realistic, time-accurate surface temperature history. Measured temperature histories ${ }^{8,9}$ from four different locations (body points 9470, 9678, 9591, and 9489) on the Space Shuttle Orbiter, as well as predicted temperature histories for three different proposed reusable launch vehicles ${ }^{4}$ (ATSpA, RLVpA, and RLV3c), were used in developing an approach for calculating an equivalent square heating pulse. It was found $^{4}$ that the maximum structural temperature was proportional to the following integral:

$$
I_{T}=\int_{t_{1}}^{t_{2}}\left(T-T_{i}\right) d t
$$

where $t_{1}$ and $t_{2}$ are the beginning and ending times of the truncated temperature history.

The time-accurate surface heating history and the equivalent square heat pulse of Shuttle Orbiter body point 9740 are illustrated in Figure 6. The equivalent square heat pulse has the same value of $I_{T}$ as shaded region of the time-accurate heating history. The timeaccurate and average values of ambient pressure for the square heat pulse are also shown.

The one-dimensional finite element model was used to calculate the maximum structural temperature for a structural thickness of $0.0032 \mathrm{~m}$ (0.125 in.) and insulator (LI-900) thicknesses of $0.0254,0.0508,0.0762$, and $0.1016 \mathrm{~m} \mathrm{(1,}$ 2,3 , and 4 in.) for each of four different heating histories. Temperaturedependent structural properties and temperature and pressure dependent insulator properties were used in the finite ele-

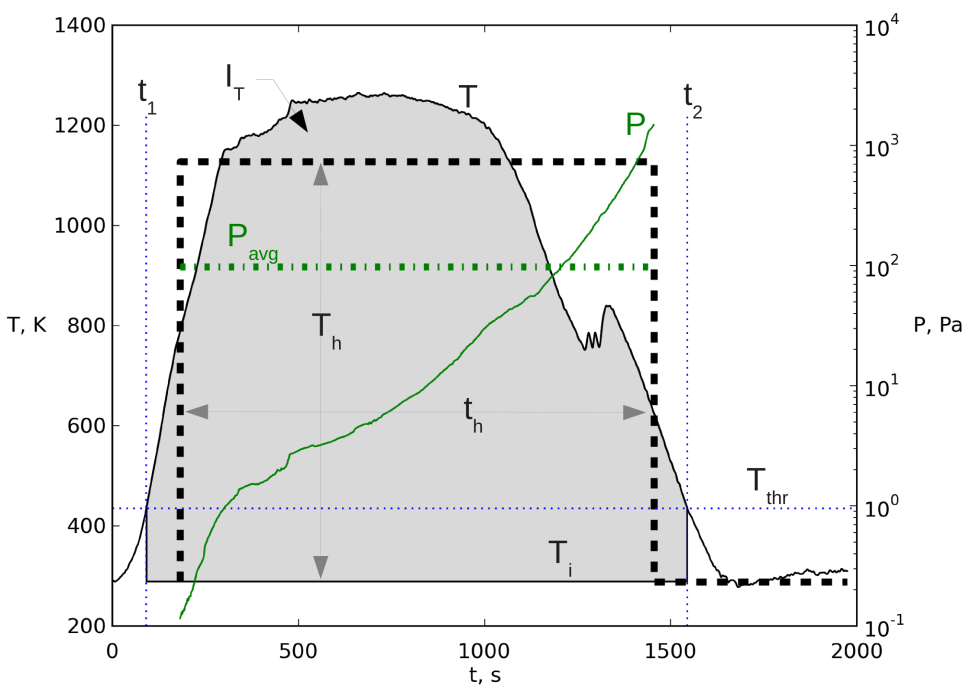

Figure 6. Simplified heating and pressure histories for BP9740 ment analyses. For comparison, the maximum structural temperature rise was calculated for each surface temperature history and geometry using the series and both approximate analytical solutions, Equations 20 and 21. However, the maximum structural temperature rise, $T_{m}$, must be known to calculate the effective constant properties. This difficulty can be readily overcome by making an initial guess and iterating to a converged solution. The series solution and the approximate solutions were each iterated independently to arrive at their respective converged solutions.

The finite element and analytical solutions for maximum structural temperature are compared in Figure 7. The figure shows the maximum structural temperature rise as a function of LI-900 tile thickness. The solid circles represent the finite element solutions, the solid lines represent the series solution, the dashed lines represent the approximate solution given by Equation 20, and the dotted lines represent the approximate solution given by Equation 21. The colors correspond to the applied surface temperature histories: black - Shuttle orbiter body point 9470, green - Shuttle orbiter body point 9489, and red - ATS reusable launch vehicle point A (windward centerline). The series solution agrees with the finite element solution to within -0.9 and 18.7 percent for the predicted maximum structural temperature rise of the cases shown on Figure 7 . The close agreement between the finite element results and the series solution tends to validate the methodology used to calculate the effective material properties and to map the transient surface temperature history to an equivalent square pulse. The two approximate equations track the series solution and the finite element well for most of the LI-900 thickness range, but start to diverge for small thickness values. 
Table 3 in the Appendix gives the detailed numerical comparisons between the finite element calculations and results using the equations developed in this paper. The table is divided into seven sections with each section consisting of results for four different insulator thicknesses for a surface temperature history identified in bold print. For each structural thickness, values of the two governing non-dimensional parameters (associated with the series solution) are given, based on the effective constant material properties and equivalent square heating pulse defined previously. The finite element prediction of the maximum structural temperature rise is given for variable material properties and a timeaccurate surface heating history. The series and approximate solutions for the

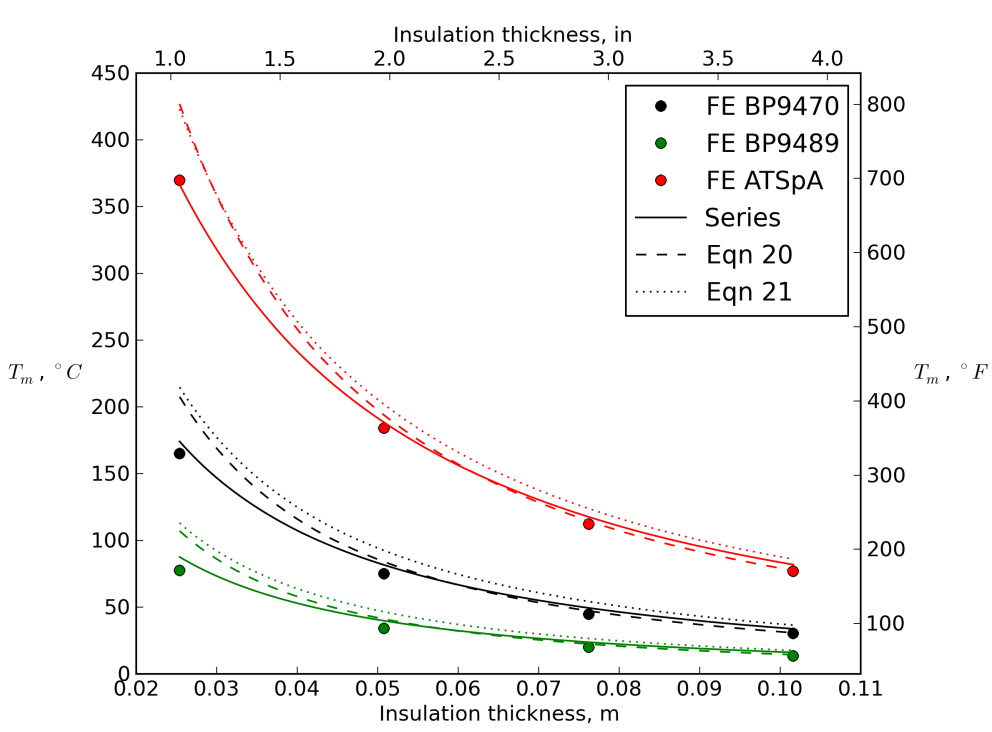

Figure 7. Maximum structural temperature rise vs insulation thickness maximum structural temperature rise are also shown, along with their errors relative to the finite element solution. The best assessment of the accuracy of using the material property simplifications combined with the surface temperature history simplifications is error associated with the series solution. The approximate solution has the same errors as the series solution combined with the approximation errors illustrated in Figure 5. The errors for the series solution range from 5.6 to 21.3 percent. The series solution is remarkably close to the finite element solution, considering the complexity of the numerical simulation and the large variation of material properties that occurs with time. For the much simpler approximate equations, Equation 20 has errors that range from 1.5 to 38.2 percent and Equation 21 has errors that range from 9.6 to 46.0 percent. The errors for the approximate solutions start to get large for relatively small values of $\gamma$, as expected from the errors shown in Figure 5. The series solution errors for the reusable launch vehicles (ATSpA, RLVpA, and RLV3c) are less than 10 percent for all calculated cases.

\section{Summary}

A simplified transient thermal problem was investigated in an attempt to gain basic insight into the thermal response of an insulated structure. A one-dimensional problem, consisting of a homogeneous insulator in perfect contact with an underlying, perfectly insulated structure was defined. From an initial uniform temperature, the outer surface of the insulator is instantaneously raised to an elevated temperature, held at that temperature for finite time, and then instantaneously returned to the initial temperature.

An analytical solution was derived for the transient response of this simplified transient problem. Although the solution is a rather unwieldy infinite series, the thermal response is completely governed by two non-dimensional parameters with physical significance. Numerical examples were plotted using properties and a heating duration representative of ceramic tiles on the Space Shuttle Orbiter.

The analytical series solution was used to calculate the maximum structural temperatures over a range of the two governing parameters. A simple function of the two governing parameters was constructed and used to approximate the maximum structural temperature over the selected range of the parameters. From this function, two approximate equations were developed for predicting the maximum structural temperature rise of an insulated structure.

Techniques were developed to choose a constant effective value for each of the temperature and pressure dependent material properties of the insulator and structural materials. A technique was also developed for defining an equivalent square heating pulse for a wide range of surface temperature histories associated with atmospheric entry. Analytical solutions for maximum structural temperature rise, using these constant effective material properties and simplified equivalent square heating pulses were compared to finite element solutions with variable material properties and time-accurate surface temperature histories for range of 
insulator thicknesses. Results for the analytical series solution were typically within 10 to 20 percent of the finite element solutions. The approximate analytical solution had similar accuracy for many of the cases studied, but began to lose accuracy as one of the non-dimensional governing parameters, $\gamma$, became small.

For the range of parameters studied, the analytical solutions developed in this paper provide a remarkably good approximation of the maximum structural temperature rise for an insulated structure subjected to atmospheric entry heating. The successful development of constant, effective values for the temperature and pressure dependent thermal properties of monolithic insulators raises the intriguing possibility that it may be possible to develop effective properties for more complex sandwich cores or composite insulations. 


\section{Appendix}

Table 3. Maximum structural temperature rise for LI900 insulation

\begin{tabular}{|c|c|c|c|c|c|c|c|c|c|}
\hline $\begin{array}{l}d_{e} \\
m\end{array}$ & $\gamma$ & $\tau_{h}$ & $\begin{array}{l}\mathrm{FE} \\
{ }^{\circ} \mathrm{C}\end{array}$ & $\begin{array}{c}\text { Series } \\
{ }^{\circ} C\end{array}$ & $\begin{array}{c}\text { error } \\
\%\end{array}$ & $\begin{array}{c}\text { Eqn } 20 \\
{ }^{\circ} C\end{array}$ & $\begin{array}{c}\text { error } \\
\%\end{array}$ & $\begin{array}{c}\text { Eqn } 21 \\
{ }^{\circ} C\end{array}$ & $\begin{array}{c}\text { error } \\
\%\end{array}$ \\
\hline \multicolumn{10}{|c|}{ BP9470 } \\
\hline 0.0254 & 0.4367 & 0.6536 & 164.86 & 174.10 & 5.6 & 207.58 & 25.9 & 214.64 & 30.2 \\
\hline 0.0508 & 0.7858 & 0.1889 & 74.84 & 81.58 & 9.0 & 83.99 & 12.2 & 92.40 & 23.5 \\
\hline 0.0762 & 1.1128 & 0.0902 & 44.74 & 49.42 & 10.5 & 47.22 & 5.5 & 54.23 & 21.2 \\
\hline 0.1016 & 1.4400 & 0.0527 & 30.33 & 33.74 & 11.2 & 30.79 & 1.5 & 36.51 & 20.4 \\
\hline \multicolumn{10}{|c|}{ BP9678 } \\
\hline 0.0254 & 0.4364 & 0.6857 & 152.83 & 173.38 & 13.4 & 206.89 & 35.4 & 213.33 & 39.6 \\
\hline 0.0508 & 0.7857 & 0.1980 & 68.76 & 81.49 & 18.5 & 84.03 & 22.2 & 92.15 & 34.0 \\
\hline 0.0762 & 1.1128 & 0.0946 & 41.05 & 49.40 & 20.3 & 47.30 & 15.2 & 54.14 & 31.9 \\
\hline 0.1016 & 1.4400 & 0.0552 & 27.81 & 33.73 & 21.3 & 30.85 & 10.9 & 36.47 & 31.1 \\
\hline \multicolumn{10}{|c|}{ BP9591 } \\
\hline 0.0254 & 0.4288 & 0.6144 & 139.86 & 155.54 & 11.2 & 186.49 & 33.3 & 193.71 & 38.5 \\
\hline 0.0508 & 0.7735 & 0.1767 & 63.32 & 72.47 & 14.4 & 74.65 & 17.9 & 82.61 & 30.5 \\
\hline 0.0762 & 1.1010 & 0.0839 & 37.78 & 43.76 & 15.8 & 41.70 & 10.4 & 48.20 & 27.6 \\
\hline 0.1016 & 1.4290 & 0.0488 & 25.58 & 29.83 & 16.6 & 27.10 & 5.9 & 32.35 & 26.5 \\
\hline \multicolumn{10}{|c|}{ BP9489 } \\
\hline 0.0254 & 0.3969 & 0.5056 & 77.47 & 87.58 & 13.0 & 107.09 & 38.2 & 113.09 & 46.0 \\
\hline 0.0508 & 0.7285 & 0.1408 & 33.92 & 39.79 & 17.3 & 41.10 & 21.2 & 46.49 & 37.1 \\
\hline 0.0762 & 1.0588 & 0.0651 & 20.08 & 23.75 & 18.3 & 22.41 & 11.6 & 26.52 & 32.1 \\
\hline 0.1016 & 1.3900 & 0.0373 & 13.55 & 16.09 & 18.7 & 14.39 & 6.1 & 17.60 & 29.8 \\
\hline \multicolumn{10}{|c|}{ ATSpA } \\
\hline 0.0254 & 0.4826 & 1.3822 & 369.70 & 366.53 & -0.9 & 426.90 & 15.5 & 423.03 & 14.4 \\
\hline 0.0508 & 0.8856 & 0.4003 & 184.05 & 188.32 & 2.3 & 193.76 & 5.3 & 201.79 & 9.6 \\
\hline 0.0762 & 1.2413 & 0.1957 & 112.23 & 117.62 & 4.8 & 114.58 & 2.1 & 123.82 & 10.3 \\
\hline 0.1016 & 1.5723 & 0.1178 & 76.97 & 81.86 & 6.3 & 77.45 & 0.6 & 85.93 & 11.6 \\
\hline \multicolumn{10}{|c|}{ RLVpA } \\
\hline 0.0254 & 0.4803 & 1.3174 & 358.76 & 348.28 & -2.9 & 405.92 & 13.1 & 403.15 & 12.4 \\
\hline 0.0508 & 0.8764 & 0.3826 & 175.04 & 177.50 & 1.4 & 182.78 & 4.4 & 190.99 & 9.1 \\
\hline 0.0762 & 1.2327 & 0.1863 & 106.19 & 110.50 & 4.1 & 107.55 & 1.3 & 116.67 & 9.9 \\
\hline 0.1016 & 1.5589 & 0.1122 & 72.75 & 76.85 & 5.6 & 72.59 & -0.2 & 80.88 & 11.2 \\
\hline \multicolumn{10}{|c|}{ RLV3c } \\
\hline 0.0254 & 0.4710 & 1.2408 & 270.03 & 276.63 & 2.4 & 324.47 & 20.2 & 323.31 & 19.7 \\
\hline 0.0508 & 0.8446 & 0.3626 & 131.45 & 139.07 & 5.8 & 143.92 & 9.5 & 151.17 & 15.0 \\
\hline 0.0762 & 1.1880 & 0.1760 & 79.73 & 86.22 & 8.1 & 84.07 & 5.4 & 91.61 & 14.9 \\
\hline 0.1016 & 1.5121 & 0.1050 & 54.48 & 59.67 & 9.5 & 56.23 & 3.2 & 63.12 & 15.9 \\
\hline
\end{tabular}

\section{Acknowledgments}

The author would like to thank NASA Langley Research Center for the opportunity to perform this work under the Floyd Thompson Fellowship. Fellowship advisors, Professors Raphael Haftka and Bhavani Sankar from the University of Florida and Dr. William Garver from Lockheed Martin provided much helpful advice and encouragement. Professor Haftka contributed essential guidance for non-dimensionalizing the governing equations and boundary conditions. Colleagues, Dr. Kamran Daryabeigi and Carl Poteet provided valuable review and discussion of the work as it progressed. 


\section{References}

${ }^{1}$ Gogu, C., Haftka, R. T., Bapanapalli, S. K., and et. al., "Dimensionality Reduction Approach for Response Surface Approximations: Application to Thermal Design," AIAA Journal, Vol. 47, No. 7, 2009, pp. 1700-1708.

${ }^{2}$ Carslaw, H. S. and Jaeger, J. C., Conduction of Heat in Solids, Oxford University Press, Oxford, 1959, Second edition.

${ }^{3}$ De Chant, L. J., "Analytical Solutions for Diffusive Finite Reservoir Problems Using a Modified Orthogonal Expansion Method," Mathl. Comput. Modelling, Vol. 28, No. 11, 1998, pp. 73-86.

${ }^{4}$ Blosser, M. L., "Analysis and Sizing for Transient Thermal Heating of Insulated Aerospace Vehicle Structures," to be published as NASA TP.

${ }^{5}$ Williams, S. D. and Curry, D. M., "Thermal Protection Materials: Thermophysical Property Data," NASA RP-1289, 1992.

${ }^{6}$ Jones, E., Oliphant, T., Peterson, P., and et.al, "SciPy: Open Source Scientific Tools for Python," URL: http://www.scipy.org, 2001-2012.

${ }^{7}$ Logg, A. and Wells, G., "DOLFIN: Automated finite element computing," ACM Transactions on Mathematical Software, Vol. 37, No. 2, 2010, pp. 28.

${ }^{8}$ Gibson, D. M. and et. al., "HYTHIRM Radiance Modeling and Image Analyses in Support of STS-119, STS-125 and STS-128 Space Shuttle Hypersonic Re-entries," AIAA Paper 2010-244, Jan. 2010.

${ }^{9}$ Zalameda, J. N. and et. al., "Application of a Near Infrared Imaging System for Thermographic Imaging of the Space Shuttle during Hypersonic Re-Entry," AIAA Paper 2010-245, Jan. 2010. 